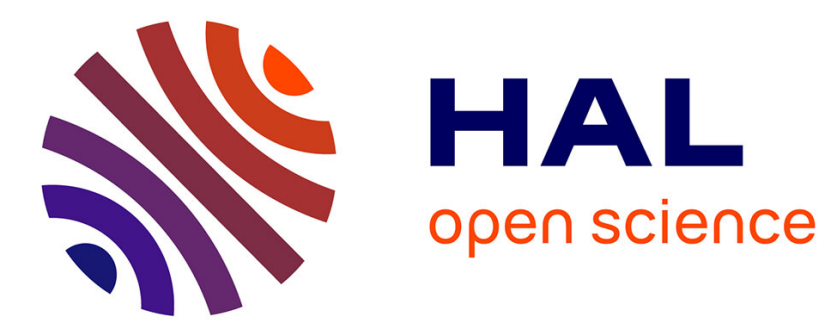

\title{
La centralité, vecteur de différence en Europe occidentale
}

Thérèse Saint-Julien

\section{To cite this version:}

Thérèse Saint-Julien. La centralité, vecteur de différence en Europe occidentale. Rey V., Saint-Julien Th. Territoires d'Europe, la différence en partage, Lyon, ENS Editions, pp.305-316, 2005. halshs00218341

\section{HAL Id: halshs-00218341 https://shs.hal.science/halshs-00218341}

Submitted on 9 May 2020

HAL is a multi-disciplinary open access archive for the deposit and dissemination of scientific research documents, whether they are published or not. The documents may come from teaching and research institutions in France or abroad, or from public or private research centers.
L'archive ouverte pluridisciplinaire HAL, est destinée au dépôt et à la diffusion de documents scientifiques de niveau recherche, publiés ou non, émanant des établissements d'enseignement et de recherche français ou étrangers, des laboratoires publics ou privés. 


\title{
La centralité, vecteur de différence en Europe occidentale
}

\author{
Thérèse Saint-Julien \\ Université Paris 1 - UMR 8506 Géographie-cités
}

\section{Résumé}

La centralité demeure en Europe occidentale un principe fort de différenciation spatiale et d'intégration territoriale. Le texte présenté évoque différentes facettes de cette contribution de la centralité à la différenciation territoriale. Il tente d'expliquer pourquoi celle-ci est restée sur le long terme un vecteur relativement stable de la transmission des différences. Quelques signes avant-coureurs d'un changement pourraient être décelés dans ce que d'aucuns interprètent déjà comme une rupture dans la production du sens de la centralité sur la durée. Rien n'interdit cependant de penser que ces évolutions qui transforment la centralité soient de nature à menacer en profondeur son rôle différenciateur.

\section{Mots clefs}

Accumulation, centralité, centralité symbolique, centre, périphérie, différence spatiale, intégration territoriale, mise en réseau des villes

Evoquer les processus territoriaux de différenciation et d'intégration en Europe invite à s'interroger sur la contribution spécifique des villes à ces dynamiques. Comment ne pas ici se souvenir que, pour toute une partie du Monde, l'invention de la ville et sa diffusion sont parties du bassin méditerranéen et que, sur la très longue durée, à partir du Moyen Age surtout, l'Europe a été le terrain d'épanouissement d'une grande civilisation urbaine. Dans cet espace, la centralité de la ville moderne s'est imposée comme un paramètre essentiel du formatage des pratiques et des représentations des territoires, devenant un principe majeur de l'aménagement de ces derniers. Les villes et les centralités qui en sous-tendent la formation et le fonctionnement y sont à la fois source de très durables régularités spatiales, et d'un renouvellement permanent de la différenciation géographique. Tout s'est donc depuis très longtemps passé comme si l'invention des modèles territoriaux spécifiques dont l'Europe a été le terrain, avait continûment intégré un regard, une expérience, et une interprétation de la ville et de sa centralité.

\section{Centres et périphéries, le poids des inégalités géographiques de l'accumulation}

Reposant sur l'individualisation de centres, la centralité urbaine est, par nature, porteuse de différenciation, participant activement à l'hétérogénéité de l'espace et des réseaux. Le centre urbain se distingue de sa périphérie par un ou plusieurs attributs (économiques, fonctionnels, politiques, symboliques...) susceptibles d'exercer une attraction - ou une domination - sur les personnes et les autres entités localisées dans sa périphérie. Lorsqu'il est amorcé, le processus d'individualisation d'un centre tend à s'auto-entretenir, au moyen de flux d'échanges dissymétriques. Ces derniers favorisent des accumulations dans le centre, au détriment de sa 
périphérie. Il s'ensuit d'importantes différences entre le centre et la périphérie. Celles-ci peuvent être quantitatives : les différentiels de masses de population, de quantités produites, de concentration d'équipements et d'institutions, de fonctions de pouvoir, sont autant de signes très tangibles des attributs du centre et des écarts creusés entre ce dernier et sa périphérie. Les différentiels centre-périphérie peuvent aussi être d'ordre qualitatif, qu'il s'agisse par exemple, du niveau de richesse, de la diversité des savoirs et des savoirs faire, des capacités collectives à innover, du degré de complexité des structures sociales et économiques, des représentations collectives de l'espace, etc..).

Tout un versant de la différenciation spatiale relève en Europe de ce principe très général. Du fait des différences géographiques qu'il engendre dans toutes les constructions territoriales, on le retrouve au cœur de très nombreuses problématiques telles que les problématiques centrepériphéries, celles relatives aux relations villes-campagnes, aux processus de polarisation et plus récemment, de métropolisation par exemple.

Ces processus d'individualisation des centres sont en outre partie prenante de la formation des dimensions hiérarchiques des structures spatiales. Accumulation et diversification, qui soustendent ces processus opèrent toujours à différents échelons géographiques. La centralité est ainsi une dimension multiscalaire de la différenciation. Compte tenu de l'ancienneté du peuplement du continent européen, les échelons auxquels se manifeste la centralité ont tendance à être plus nombreux que sur les autres continents avec en particulier, un semis beaucoup plus dense des villes petites et moyennes. Cette forte densité des centres observée en Europe est en général rapprochée de la vitesse moyenne de communication au moment de la mise en place des grandes lignes du réseau urbain européen, c'est à dire des grandes phases de l'appropriation du territoire par les sociétés européennes. A ces propriétés multiscalaires sont associées non seulement des formes plus ou moins hiérarchisées de concentration, mais aussi des systèmes de relation imbriqués, des territoires plus ou moins emboîtés, et surtout des systèmes de concurrence spatiale souvent profondément ancrés dans le territoire. D'un bout à l'autre de l'Europe occidentale on discerne bien ces chaînes de centres relativement hiérarchisées et les aires emboîtées de leur polarisation, territoires plus ou moins pris en tenaille dans un système de concurrences interurbaines relativement denses.

Des dimensions plus spécifiques de l'individualisation des pôles peuvent conforter celles directement liées à la centralité ordinaire. Ces dimensions sont celles de la spécialisation des centres. Toutes choses égales quant à la position de ces derniers dans un système de lieux centraux, leur force réside dans la nature de leur spécialisation. Ces spécialisations ont depuis longtemps distingué les villes entre elles, et construit de véritables identités urbaines, beaucoup plus durables en général que les spécialisations elles-mêmes. Combinées aux fonctions centrales proprement dites, elles contribuent à définir le poids économique des villes, leur structure sociale, leur position relative dans un ensemble régional, national et désormais, européen, leur force de territorialisation, leurs capacités d'innovation. Or ces fonctions plus spécifiques, qui reposent sur des faisceaux d'interdépendances géographiques moins exclusivement tributaires de la continuité spatiale que de la connexité des lieux, engendrent des formes différentes de polarisation. Les interdépendances liées aux spécialisations des centres ont pour effet d'une part, de surclasser ou de déclasser les villes par rapport aux niveaux et aux qualités des fonctions de la centralité ordinaire qu'elles exercent et d'autre part, de rendre beaucoup plus complexes les système d'interrelation des villes entres elles. Cette dimension plus spécifique de l'individualisation des pôles urbains contribue beaucoup à accroître la réticularité des réseaux de villes, soit en court-circuitant des 
La centralité, vecteur de différence en Europe occidentale. In Rey V., Saint-Julien Th. (dir), 2005, Territoires d'Europe la différence en partage, Lyon, Editions de l'ENS-LSH, 305-315.

niveaux hiérarchiques, soit en développant les relations entre niveaux équivalents. De ce fait les villes peuvent s'éloigner d'un ordonnancement spatial multiscalaire strictement hiérarchisé, ce qui n'a pas manqué de jouer durablement en Europe dans le sens de l'accentuation des différenciations inter-régionales.

Différence et intégration sont ici inséparables. Les différences engendrées par les polarisations urbaines sont territorialement intégratrices. Aux différents échelons géographiques, la construction de totalités territoriales différentes s'appuie sur la force des liens tissés entre centres, et entre centres et périphéries. Cette force est une des dimensions essentielles de la matrice des organisations régionales en Europe occidentale.

\section{Centralité et diversité des formes de structuration régionale}

On trouve en Europe occidentale plusieurs expressions régionales bien individualisées de ce double rapport de la centralité à la différence et à l'intégration territoriale. On choisit d'illustrer cette dualité en considérant les dynamiques qui ont, à échelle moyenne, engendré différents modèles de construction régionale en Europe. Echelons d'intégration relativement robustes, ces structures régionales, se sont très progressivement construites en Europe occidentale, et par la suite pérennisées, selon des modèles que différencient le poids des pôles, leur structuration hiérarchique, la portée géographique de leurs interdépendances, en relation avec les échelons auxquels a durablement joué la concurrence interurbaine.

D'un bout à l'autre de cette Europe occidentale et sur le très long terme, la dynamique différenciation/intégration liée à la centralité s'est développée dans des contextes suffisamment différents pour que s'édifient plusieurs modèles de structuration régionale. Leurs spécificités sont en premier lieu à rapprocher des inégalités des densités de population et d'activité (espacement des villes de plus de $10000 \mathrm{~h}$ de $7 \mathrm{~km}$ aux Pays-Bas à $30 \mathrm{~km}$ en Irlande). Celles-ci ont contribué par exemple à structurer la dorsale rhénane (Flandre, Rhénanie, Plaine du Pô), individualisant cette région de celles situées à l'ouest, au sud et à l'est. Ces spécificités sont à rechercher en deuxième lieu dans les types de développement urbain privilégiés durant les phases de montée en puissance des villes (villes vivant plutôt de la rente rurale dans l'Europe du sud-ouest, villes marchandes flamandes, villes princières rhénanes, villes industrielles de la Rhénanie et de l'Italie du Nord, villes touristiques littorales où montagnardes, etc.). Elles s'inscrivent alors dans les combinaisons qui, au fil des temps, se sont tissées dans le réseau urbain régional entre les fonctions liées à la centralité ordinaire et celles, plus spécialisées, porteuses de centralités spécifiques. La diversité contemporaine de ces constructions renvoie enfin aux conditions de la mise en concurrence des centres les uns par rapport aux autres en relation à la fois, avec la dimension et le type de l'entité étatique à l'intérieur de laquelle s'est déployée cette mise en concurrence, et avec les caractéristiques de l'espace-temps durant la période clé de mise en place de ces réseaux.

Analysant la structuration régionale de l'Europe occidentale continentale, E. Juilliard (1976) avait attiré l'attention sur le rôle tenu par les réseaux urbains dans ces structurations et identifié les types principaux d'articulation. Cattan et al. (2000) ont repris ces types en en redéfinissant, la fin du $\mathrm{XX}^{\mathrm{e}}$ siècle, les contenus et l'expansion spatiale. En s'en tenant aux échelons des plus grandes villes, on peut ainsi distinguer quatre grands types. Le type rhénan s'individualise par un grand nombre de grandes villes fortement concurrentes, têtes de réseaux régionaux aux niveaux de fonction faiblement différenciés, avec des aires d'influence réduites et de dimension comparable, et ayant en général résisté durant toute la période moderne à la 
formation d'Etats centralisés. Ce type caractérise principalement la région de la dorsale des forts potentiels démographiques et économiques qui, en Europe, prend en écharpe Flandre, Rhénanie. On peut identifier en Italie du nord-est, dans les régions alpines, en Lorraine, dans le Levant espagnol et en Bohème, un type de structuration régionale par les villes assez voisin du précédent. Ce type " intermédiaire dominant " s'accommode cependant d'une puissance des centres majeurs en moyenne plus faible et, dans les deux derniers cas, d'espaces polarisés plus étendus, du fait de densités de population beaucoup plus faibles. A l'opposé, le type parisien est repérable autour Paris en France, de Dublin en Irlande, de Lisbonne au Portugal, d'Athènes en Grèce, de Vienne en Autriche ou de Budapest en Hongrie, voire de Londres en Grande Bretagne. Il correspond bien à des structures régionales dans lesquelles le poids relatif d'un pôle principal, souvent élevé au rang de capitale d'Etat, est suffisamment démesuré pour que, dans sa mouvance directe, la marge laissée au développement de centres de taille intermédiaire ait été très faible, ce qui conduit à une exacerbation régionale de la dissymétrie centre périphérie. Un quatrième type peut être considéré comme une forme affaiblie du type précédent. Avec le type périphérique dominant dans le sud-ouest de la France, l'Italie du sud, l'est de Allemagne, la métropole régionale a pu se développer à une distance relativement grande de la très grande métropole nationale; elle est un centre dispensateur de services de bon niveau pour une aire relativement étendue mais dont les marges sont parfois incomplètement intégrées. Ce modèle a pu aussi se développer dans l'Europe centrale et balkanique avec cependant, un gradient centre périphérie un peu plus faible. Ici, des politiques correctrices conduites par les régimes socialistes durant une quarantaine d'années qui visaient, par la démultiplication des chefs-lieux administratifs relais obligés de toutes les structures politico-administratives, à limiter cette source de différence spatiale jugée incompatible avec les principes même de la territorialité socialiste (Rey 1997).

Les spécificités de ces modèles régionaux de la centralité ne peuvent se comprendre que replacés dans le temps long de l'histoire européenne. Elles révèlent la permanence des formes de la centralité en tant qu'expression de la différenciation et de la structuration de l'espace géographique. Elles traduisent aussi la capacité de ces formes à intégrer les changements et à en restituer très durablement les effets. Elles contribuent à donner une lisibilité territoriale très forte à la diversité des organisations socio-politiques et des cultures du territoire. Elles concourent enfin, à engendrer des images emblématiques qui resserrent les liens de territorialité autour des centres.

\section{Mise en réseau des villes et valorisation des différences dans le processus d'intégration territoriale}

Une question resurgit, ravivée chaque fois qu'un pas significatif est franchi dans un processus d'intégration territoriale. La centralité n'est-elle pas porteuse de sa propre limite? L'intégration qu'elle engendre à différents échelons géographiques, n'irait-elle pas à l'encontre de la conservation d'architectures territoriales différenciées ? Dans un contexte d'accentuation des processus d'intégration territoriale, la centralité ne garde toute sa force que si l'intégration ne conduit pas à un progressif effacement des différences, si ces dernières conservent une force de renouvellement, suffisante pour que différences et intégration s'épaulent mutuellement. On voudrait montrer ici comment, à partir de l'exemple des grands pôles urbains, des centralités se redessinent aujourd'hui en se réappropriant la force de différences qui se sont structurées sur le long terme à l'échelon européen.

On note en premier lieu que l'intégration croissante du territoire européen s'accompagne de la formation d'une structure centrale polycentrique qui repose à la fois, sur la diversité des pôles 
urbains majeurs à l'échelon de ce vaste ensemble et sur la multiplicité de leurs appartenances. Ce centre polycentrique se distingue de la grande région urbaine constituée par la mégalopole rhénane, dont la cohésion est faite à la fois de caractéristiques morphologique et fonctionnelles et d'une forte intégration territoriale, l'ensemble formant une structure relevant de ce type rhénan décrit précédemment. En effet, cette région, axe de très fort potentiel humain et économique, n'est cependant pas à proprement parler le centre, même si elle s'y rattache pour partie. Le centre proprement dit, polycentrique par construction, est un véritable réseau constitué par un nombre limité de métropoles pas très éloignées, et en étroite relation les unes avec les autres. Londres et Paris en sont de fait, les piliers. Au-delà, selon les cadres dans lesquels on se place, le centre peut intégrer, Amsterdam, Bruxelles, Francfort, et Zurich. Berlin rejoindra-t-elle prochainement cette structure centrale ? Londres et Paris exceptés, ces pôles ne sont pas les plus grandes villes de l'ensemble européen, mais les plus grandes dans leur système urbain national respectif, La mégapole et la structure centrale polycentrique ont en commun certains nœuds tels Amsterdam, Bruxelles et Francfort. Au total, ce niveau du centre d'un système des villes européennes en voie d'intégration forte, véritable cœur du continent, se construit sur une base polycentrique qui intègre pleinement les spécificités et les potentialités de chacune de ces métropoles.

Depuis une quinzaine d'années, l'intensification des échanges entre les villes du centre a certes progressé relativement plus vite qu'entre les autres grands pôles urbains, ce qui a beaucoup conforté la cohésion de cette structure centrale. Pourtant, un autre fait majeur, et plus nouveau peut-être, est le développement des relations entre les très grandes villes plus périphériques des pays du sud et de l'est en particulier. Celles-ci sont le plus souvent des capitales d'Etat, mais parfois aussi de grandes métropoles économiques. En consolidant leur arrimage au centre d'une part, et en les insérant d'autre part dans le réseau plus large de l'ensemble des villes "capitales", cette intensification a progressivement modifié leur position relative dans l'espace européen. Elle représente un nouveau niveau d'intégration. Celui-ci pourrait à terme contribuer à atténuer un peu la forte hiérarchisation qu'impose à l'ensemble de l'espace européen un centre dont la structure, bien que polycentrique, s'inscrit intégralement dans la région du nord-ouest traditionnellement la plus riche et la mieux connectée. Au total, ce réseau de l'ensemble des villes " capitales", en position intermédiaire et aujourd'hui en cours de consolidation, constitue un vecteur très dynamique de la mise en relation des villes à l'échelon du continent.

Pourtant, à court et probablement à moyen terme, les réseaux nationaux de villes demeurent l'échelon le plus cohérent et le plus structuré du système des villes européennes. Ce maintien n'est en contradiction ni avec la formation d'une structure centrale polycentrique, ni avec l'intensification de la mise en réseau des "villes capitales". Il n'est pas non plus incompatible avec une croissance plus généralisée à l'échelon européen des interdépendances entre les villes. Dans un premier temps, les échanges internationaux des villes ont tendance à passer largement par le petit nombre des relais que constituent les plus grandes villes. Dans un processus international d'intégration, ces dernières jouent souvent le rôle de têtes de pont, bénéficiant d'une polarisation incontestée sur leur territoire national. En retour ce processus d'intégration territoriale apparaît d'autant plus actif que les systèmes urbains nationaux sont eux-mêmes plus intégrés et que l'accès aux réseaux internationaux est facilité par l'existence d'un petit nombre de relais efficaces pour un même territoire. A peu près partout en Europe, la reprise des concentrations métropolitaines est un des signes de la vigueur de ces circuits hiérarchisés de l'intégration territoriale, et de la vigueur des dynamiques propres aux échelons régionaux et nationaux qui, au moins en partie, les sous-tendent encore. 
Enfin, en s'internationalisant, les réseaux interurbains spécialisés, avant tout définis par une logique de production de biens ou de services, font qu'une part des relations interurbaines s'affranchit de contraintes liées tant à l'organisation hiérarchique des systèmes nationaux qu'à la distance. Tout en contribuant à renouveler les bases des polarisations urbaines, ces réseaux démultiplient ainsi les articulations entre systèmes urbains nationaux et structure centrale, et aussi entre systèmes urbains nationaux.

Au total, la dynamique contemporaine d'intégration du réseau des villes européennes joue sur plusieurs registres, qui valorisent à la fois différenciation et intégration territoriale. La logique relationnelle apparaît doublement intégrative dans la mesure où elle opère à différents échelons géographiques, et où elle joue à la fois sur les effets de la dépendance liée à la proximité, dans l'espace et dans les réseaux. Liée à la proximité, cette logique relationnelle reste, un facteur fort de la cohésion du réseau des villes européennes. D'une part, dans un contexte général d'élargissement de la portée géographique des relations, les freins de la distance demeurent suffisamment importants pour que, à l'échelon de l'ensemble du continent, les gradients centre-périphérie constitués autour des villes continuent de jouer un rôle très actif. D'autre part, la forte cohésion des systèmes urbains nationaux, qui s'appuie grandement sur les exigences de la continuité de la desserte, continue aussi de dépendre au moins pour partie de cette même logique de proximité. De son côté, la logique relationnelle de connexité qui, en jouant seule, aurait pu avoir tendance à renforcer la concentration sur quelques nœuds urbains principaux, a été partiellement freinée par la force des structures en place dans le contexte de réseaux urbains anciens et relativement stables. De son côté, la logique de ressources appelle en permanence une mise en valeur différenciée des lieux, et donc un renouvellement permanent des spécialisations urbaines et régionales. Enfin, une logique de maillage impose l'intégration de fortes différences infra continentales, en particulier celles des organisations nationales de la centralité, conservant ainsi à cette dernière sa fonction de différenciation dans l'intégration.

\section{La centralité dans la transmission des différences sur le long terme}

On a vu que, dans son principe même, la centralité est porteuse de différence. A partir de l'exemple des constructions régionales en Europe, ou de celui de l'ébauche d'une construction urbaine de dimension continentale, on a aussi noté que la centralité contribuait de manière très active et sur le long terme à conserver, en les intégrant, les traces de rapports parfois très anciens que les sociétés ont entretenu avec leur espace. Les villes sont apparues à la fois enracinées dans une culture, modelées par une structure sociale, un contexte régional et fortement soumises aux aléas de l'histoire et aux fluctuations de l'économie et de la démographie. On peut être frappé par le forte " inertie " du réseau urbain européen sur le long terme, surtout pour les villes occupant les niveaux les plus élevés de la hiérarchie. Il n'a pas fallu moins de 5 siècles pour déplacer le centre de gravité de Venise à Londres. Les deux cinquièmes des villes de plus de 10000 habitants vers 1800 avaient déjà plus de $10000 \mathrm{~h}$ en 1500 , et le tiers des 835 villes de plus de 50000 habitants aujourd'hui étaient les plus grandes villes européennes de 1800 . Le phénomène apparaît de manière particulièrement exemplaire dans l'Europe méditerranéenne, moins "chahutée" par la révolution industrielle. Il y a lieu de s'interroger sur les processus qui, sur le long terme, font de la centralité un vecteur particulièrement efficace à la fois de la diffusion du changement et de la transmission des différences géographiques. Parmi les processus les plus actifs, trois méritent ici de retenir l'attention. 
Les relations très étroites que la centralité entretient avec l'innovation sont une première clef pour comprendre renouvellement et transmission des différences. Deux forces, contradictoires en apparence seulement, interviennent. D'une part les pôles urbains, les plus grands en particulier, sont des lieux privilégiés d'invention du changement, de captation des innovations, puis de diffusion. Cette position leur assure non seulement un bon renouvellement des bases de leur distinction mais aussi souvent l'élargissement de ces bases, ce qui ne manque pas d'améliorer leur position relative dans le réseau. Ce faisant, pour un territoire donné, les grands centres sont les lieux emblématiques de la capacité d'une société à inventer son futur. A partir de ces pôles principaux d'autre part, la propagation des innovations se fait en général suivant le sens descendant de la hiérarchie urbaine, ce qui définit ainsi des directions privilégiées de la réactivité des territoires. Cependant, les concurrences entre centres urbains jouant dans le sens d'une accentuation des vitesses de propagation, les effets cumulatifs dont pourraient bénéficier sur la durée les centres ayant les premiers capté l'innovation peuvent être freinés. Les gradients initiaux intégrés dans le changement ont donc tendance à se pérenniser, au plus à s'accentuer. Ainsi par exemple, les villes qui le long des grands itinéraires commerciaux entre Mer du Nord et Méditerranée, ont pu résister au modèle des Etats nations jusqu'au cœur du XIX ${ }^{\mathrm{e}}$ siècle, ont pu le faire en intégrant ce modèle, jouant l'un contre l'autre les Etats rivaux, ou formant des alliances entre elles, voire cherchant des annexions territoriales (Provinces Unies de Hollande, Florence). L'exemple de la diffusion de la révolution industrielle est tout aussi riche d'enseignements. Cette révolution a certes suscité des villes nouvelles, accéléré le développement des grands ports et renforcé la force démographique et économique des plus grands pôles. Cependant, avec la rapidité de plus en plus grande de la diffusion de cette révolution, les déformations interurbaines ont été plus limitées dans les pays plus tardivement atteints. Ainsi, plus précoce en Grande-Bretagne et en Belgique, cette révolution y a apporté des perturbations géographiques relativement grandes. Plus tardive en Allemagne et en France, et a fortiori en Italie et en Espagne, venue après le développement des chemins de fer, elle y a plus systématiquement épousé les formes et les dynamiques des réseaux urbains pré-industriels, introduisant ainsi de moindres bouleversements.

Une deuxième clé doit être introduite. On a évoqué la dimension intégrative de la centralité, son rôle dans la cohésion des territoires. A elle seule, l'intégration par la centralité ne saurait cependant suffire à fonder un processus de territorialisation. Intégration et territorialisation se confortent à la condition que, sur la longue durée, la centralité reste une expression forte des attaches d'un groupe à une partie de l'espace. Il y a territorialisation quand la centralité construite a pu s'inscrire dans les mémoires collectives auxquelles elle donne un cadre durable, des dimensions matérielles et sensuelles, des images spatiales. Ces derniers viennent, à tout instant, raviver les mémoires de pratiques passées, donner des repères collectifs à différents ordres de transaction de la vie ordinaire, qu'il s'agisse par exemple de transactions politiques, économiques, ou religieuses, dessiner des cadres pour le futur. Cette inscription territoriale des transactions sociales dans la centralité apparaît relativement incontournable et ce d'autant qu'en retour, la centralité ainsi construite modèle représentations et symboles auxquels elle contribue à donner une existence tangible. Par l'appropriation collective des lieux qu'elle engendre, la centralité peut être lue comme une composante forte de la territorialité, et la ville-centre comme emblème du territoire revendiqué. On comprend alors aisément que plus la profondeur historique de cette appropriation collective est grande, plus la centralité est partie prenante des mémoires vives. 
Enfin, la stabilité de ce principe de différenciation territoriale est à rechercher dans une longue tradition du réinvestissement des mêmes lieux. Souvent considéré comme un trait majeur de la culture européenne de l'espace en général, et de la ville en particulier. De très nombreuses observations vont dans ce sens. L'idée, fréquente auprès des architectes et des urbanistes (Choay 1994, Huet 1998), est souvent reprise par des historiens et des géographes. Le fait est spectaculaire en Europe dans le remodelage pluriséculaire des espaces urbanisés. On a remodelé et réinvesti les mêmes lieux plus qu'on n'a créé de villes nouvelles. L'idée a constamment prévalu qu'une ville se reconstruit en permanence sur elle-même et dans la continuité. La ville de l'occident européen a, sur la longue durée, fonctionné comme un puissant instrument de recyclage des valeurs symboliques du passé, pour une projection sur l'avenir. Cette nécessité de la continuité comme principe de légitimation, qui correspond à une manière bien particulière de vivre la ville, est en rupture par rapport à ce qui a pu être noté ailleurs, par exemple en Asie et plus particulièrement en Inde. Ici, chaque dynastie nouvelle légitimait son pouvoir en abandonnant la ville de la dynastie précédente et en construisant une ville neuve. A bien des égards, l'urbanisme est apparu en Europe comme une reconstruction de l'histoire comme un récit urbain. L'exemple du développement technopolitain récent peut illustrer ce rôle de la transmission de la différence par les villes. Les technopôles ou parcs technologiques se sont diffusés dans toute l'Europe occidentale dans les années 80-90. Or, on observe, qu'en général, ces créations ne se sont pas développées de manière diffuse (quelques exceptions comme le corridor le long de l'autoroute LondresBristol) mais qu'elles se sont greffées sur des villes dont le potentiel était déjà important, contribuant à diversifier et à renouveler les bases de ces dernières, sans imposer de véritables discontinuités dans les mémoires des lieux. A l'échelon interurbain, les réinvestissements sont plus spectaculaires encore. Les cas de déplacement de centre de gravité des réseaux sont très rares, et surtout très lents quand ils existent. Les effets sur le système des villes européennes en cours d'intégration de la remise à niveau de Berlin présente un cas d'école particulièrement intéressant à suivre dans les années qui viennent.

\section{Conclusion}

On a tenté de montrer comment la centralité demeurait en Europe un principe fort à la fois de différenciation spatiale et d'intégration territoriale. On a aussi cherché à expliquer pourquoi elle restait sur le long terme un vecteur relativement stable de la transmission des différences. Quelques signes avant-coureurs d'un changement pourraient être décelés en Europe dans ce que d'aucuns interprètent déjà comme une rupture dans la production du sens de la centralité sur la durée. On songe, par exemple, à l'affaiblissement des structurations multiscalaires, dans le cas où les relations en connexité prendraient le pas sur les relations en continuité, à l'effacement, ou tout au moins, à la moindre lisibilité des connexions entre présence des villes et structuration des territoires, la relative perte de l'expérience physique de la centralité, et enfin les tentations d'une certaine muséification de cette dernière, dans les villes en particulier. Rien n'interdit cependant de penser que ces évolutions qui transforment la centralité soient de nature à menacer en profondeur son rôle différenciateur.

\section{Références}

Brunet R., 1989, Les villes « européennes ». Datar/Reclus, La Documentation Française.

Brunet R., 1996, L'Europe des réseaux, in Pumain D., Saint-Julien Th. (ed.), Urban Networks in Europe, John Libbey Eurotext-INED, 131-150.

Camagni R., 1993, Organisation économique et réseaux de villes, in P.-H. Derycke (dir.), Espace et 
La centralité, vecteur de différence en Europe occidentale. In Rey V., Saint-Julien Th. (dir), 2005, Territoires d'Europe la différence en partage, Lyon, Editions de l'ENS-LSH, 305-315.

dynamiques territoriales, Economica.

Cattan N., Pumain D., Rozemblat C., Saint-Julien Th., 1994, Le système des villes européennes. Paris, Economica, 243 p.

Cattan N., Saint-Julien Th., 1998, Vers un modèle métropolitain intégré en Europe occidentale. L'Espace géographique, $\mathrm{n}^{\circ} 1,1-10$.

Choay F., 1994, Le règne de l'urbain et la mort de la ville. In La ville art et architecture en Europe. Centre G. Pompidou, 26-35.

Choay F.,1980, La règle et le modèle. Paris, Seuil, 1980.

Dematteis G., 1996, Towards a Unified Metropolitan Urban System in Europe, in Pumain D., SaintJulien Th. (ed.), Urban Networks in Europe, John Libbey Eurotext-INED, 19-28.

Huet B., 1998, Une génétique urbaine. Urbanisme, n 303, 56-61.

Lepetit B., Pumain D., 1993, Temporalités urbaines. Paris, Anthropos.

Pumain D., Saint-Julien Th. (ed.), 1996, European Urban Network. John Libbey Eurotext limited,

Pumain D., Saint-Julien Th., Van Der Haegen (ed.), 1991, Cities in movement. Urban systems on the eve of the $21^{\text {st }}$ century. Acta Geographica Lovaniensa, 31, 230 p.

Roncayolo M., 1980, La ville et es territoires. Paris Gallimard, Folio.

Rey V., 1997, Europes orientales. In Brunet R., Rey V. (dir.), Europes orientales, Russie, Asie Centrale. Géorgaphie Universelle, Tome 10, Livre 1 ${ }^{\mathrm{er}}$, Belin-RECLUS, 8-206.

van den Berg L., Drewett R., Klassen L.H., Rossi A., Vijverberg C.H.T., 1982, A Study of Growth and Decline, Urban Europe, vol. 1, Pergamon Press. 